\title{
Aberrant CD20 Expression in Angioimmunoblastic T-cell Lymphoma
}

\author{
Takayoshi Tachibana ${ }^{1,2}$, Naoto Tomita ${ }^{1}$, Mitsuko Furuya ${ }^{3}$, Shoji Yamanaka ${ }^{3}$, \\ Kengo Takeuchi ${ }^{4}$, Naoya Nakamura ${ }^{5}$, Hiroyuki Fujita ${ }^{1}$ and Yoshiaki Ishigatsubo ${ }^{1}$
}

\begin{abstract}
We report on a 60-year-old man, having angioimmunoblastic T-cell lymphoma (AITL) with aberrant expression of CD20. The initial biopsy specimen showed features of AITL with the typical $\mathrm{CD}^{+}{ }^{+} \mathrm{CD} 20^{-}$immunophenotype. As the disease progressed, biopsy specimens of the recurrent lesions showed unusual results for AITL with the CD20 $0^{+}$immunophenotype in both flow cytometry and immunohistochemistry analysis. Double immunostaining confirmed that the lymphoma cells were simultaneously positive for CD3 and CD20. The disease became resistant to combined chemotherapies and the patient died 3 years after the initial diagnosis. Autopsy revealed a composite of AITL and EBV-positive diffuse large B-cell lymphoma. Although it is unknown whether the instability of the CD20 antigen expression is correlated with the behaviour of AITL, the administration of rituximab should be considered as an alternative therapeutic option in such cases.
\end{abstract}

Key words: angioimmunoblastic T-cell lymphoma, CD20, rituximab, flowcytometry, immunohistochemical staining, EBER in situ hybridization

(Intern Med 50: 495-499, 2011)

(DOI: 10.2169/internalmedicine.50.4386)

\section{Introduction}

Angioimmunoblastic T-cell lymphoma (AITL), a frequent subtypes of peripheral T-cell lymphoma, is associated with a poor prognosis and a 5-year survival rate of $30-36 \%(1,2)$. Common clinical manifestations include systemic lymphoadenopathy, hepatosplenomegaly, fever, anemia, skin rash and polyclonal gamma-globulinemia. Involved lymph nodes show polymorphous infiltrates including $\mathrm{T}$ cells with clear cytoplasm, proliferation of arborizing high endothelial venules (HEV), and proliferation of follicular dendritic cells. B-immunoblasts infected with Epstein-Barr virus (EBV) are also usually present in AITL. Rearrangements of T-cell receptor genes are detectable in about $70 \%$ of patients with AITL. In addition, rearrangement of the immunoglobulin heavy chain $(\operatorname{IgH})$ gene is sometimes detectable due to the expanded EBV-positive B-cell population (3). The lym- phoma cells of AITL are commonly positive for T-cell markers, such as $\mathrm{CD} 2, \mathrm{CD} 3$, and $\mathrm{CD} 5$, and negative for $\mathrm{B}$ cell markers, such as CD20 and CD79a (4). CD4 and CD10, the postulated normal counterparts of follicular helper Tcells, are also expressed (5). More than 20 cases of CD20positive T-cell lymphomas have been reported to date (6). These cases include peripheral T-cell lymphoma, T-cell large granular lymphocytic leukemia, T-cell prolymphocytic leukemia, and anaplastic large cell lymphoma. In the current report, we present a rare case of AITL with aberrant CD20 expression on AITL cells.

\section{Patient and Methods}

A 60-year-old man presenting with cough, fatigue, and appetite loss was admitted to our hospital in January 2006. Physical examination revealed icteric eyes, systemic lymphadenopathy (cervical, axillary, and inguinal), skin eruption on

\footnotetext{
${ }^{1}$ Department of Internal Medicine and Clinical Immunology, Yokohama City University Graduate School of Medicine, Japan, ${ }^{2}$ Department of Hematology, Kanagawa Cancer Center, Japan, ${ }^{3}$ Department of Pathology, Yokohama City University Graduate School of Medicine, Japan, ${ }^{4}$ Department of Pathology, Cancer Institute, Japanese Foundation for Cancer Research, Japan and ${ }^{5}$ Department of Pathology, Tokai University School of Medicine, Japan

Received for publication August 18, 2010; Accepted for publication November 12, 2010

Correspondence to Dr. Naoto Tomita, cavalier@ch-yamate.dlenet.com
} 
the posterior chest, and mild leg edema. The pathology of the inguinal lymph node biopsy specimen was consistent with AITL. Bone marrow involvement was not detected. The patient underwent 8 courses of THP-COP [pirarubicin (THP-ADR), cyclophosphamide (CPA), vincristine (VCR), and predonisolone], with the first complete remission (CR) achieved in February 2006. In October 2006, systemic lymphadenopathy reappeared, and an axillary lymph node biopsy was performed. Pathological analysis confirmed the recurrence of AITL. After 3 courses of ESHAP (etoposide, methylprednisolone, cytarabine [Ara-C], and cisplatin), the second CR was achieved. This was followed by autologous peripheral blood stem cell transplantation in March 2007. The patient remained in a stable phase for 10 months, until the second relapse, which occurred in January 2008. After the administration of 2 courses of hyper-CVAD/MA (THPADR, CPA, VCR, methotrexate, and Ara-C), the third CR occurred.

In January 2009, the patient experienced an episode of hemorrhagic colitis. Examination by fiber-optic colonoscopy detected a bleeding ulcer at the ileocecal colon. Pathologic examination of the ulcer biopsy specimen strongly suggested the third relapse of AITL. Immune-compromised infections such as adenoviral hemorrhagic cystitis and pneumonia occurred following the third relapse. In February 2009, the patient died from a refractory hemorrhage and an autopsy was performed.

\section{Immunohistochemistry and in situ Hybridization}

Mouse monoclonal antibody against CD20 and rabbit monoclonal antibody against CD3 were purchased from Dako (Carpinteria, CA, USA). Immunohistochemistry was done using Envision Kit (Dako) and autoclave antigen retrieval. Working dilution was $1: 100$, respectively. As negative controls, the primary antibodies were replaced by mouse or rabbit $\mathrm{IgG}$, respectively. As the positive controls for these antigens, normal lymph nodes were used. Alexa Fluor 555 goat anti-rabbit IgG and Alexa Fluor 488 goat anti-mouse IgG (Invitrogen, San Diego, CA, USA) were used for double staining, according to the methods previously described (7). In situ hybridization for EBV was done using EBV (EBER) PNA Probe and PNA ISH Detection kit (Dako) according to the manufacturer's instructions.

\section{Morphology, Flow Cytometry, Immunohistochemistry, and Karyotype}

\section{First biopsy: (AITL with $C D 3^{+} C D 20$, inguinal lymph node, in January 2006)}

On Haematoxylin and Eosin (HE) staining, the biopsy specimen showed a diffuse infiltration of atypical $\mathrm{T}$ cells with clear cytoplasm, accompanied by marked proliferation of HEV (Fig. 1a). Immunohistochemical analysis showed these neoplastic lymphocytes to be CD20 negative and CD3 positive (Fig. 1b, c). An irregular network of $\mathrm{CD} 21^{+} 35^{+}$follicular dendritic cells surrounding the HEV was also noted. Flow cytometric and karyotypic analysis were not available due to the limited amount of the biopsy material.

\section{Second biopsy: (AlTL with $\mathrm{CD3}^{+} \mathrm{CD20}$, axillar lymph node, in October 2006)}

The biopsy specimen showed diffuse infiltrating $\mathrm{CD}^{+}$ CD20 neoplastic lymphocytes with clear cytoplasm. CD35 follicular dendritic cells were detected along with HEV. These findings were consistent with recurrent AITL. Results of flow cytometric analysis of CD45 gating (Fig. 2a) also supported the notion that these lymphocytes were $\mathrm{CD}^{+} \mathrm{T}$ cell lymphoma cells (Fig. 2b). Consistent with the typical pattern seen in AITL, the cells also were CD20 negative (Fig. 2c). The expression of other surface markers was as follows; $\mathrm{CD}^{+}, \mathrm{CD}^{+}, \mathrm{CD}^{+}, \mathrm{CD} 7, \mathrm{CD} 8, \mathrm{CD}^{-} 0^{+}, \mathrm{CD} 19^{-}, \kappa$ and $\lambda$ (data not shown). Karyotypic analysis showed the following abnormality; 50,XY,+X,+5,-19,+mar1,+mar2,+mar 3[2]/51,idem,+18[1].

\section{Third biopsy: (AITL with $\mathrm{CD}^{+} \mathrm{CD20}^{+}$, cervical lymph node, in January 2008)}

The biopsy specimen showed diffuse proliferation of recurrent lymphoma cells on HE staining. These tissues exhibited a similar morphology to that of the first and the second biopsy tissues; i.e., cells showed the enlarged nuclei with clear cytoplasm typical of AITL cells (Fig. 1d). However, immunohistochemical analysis revealed that many of the lymphoma cells were positive for CD20 (Fig. 1e) as well as for CD3 (Fig. 1f). Immunofluorescent double staining revealed the co-localization of CD20 with CD3 in these cells (Fig. 1g, h, i). Flow cytometric analysis also revealed an unusual pattern of surface marker expression. In the flow cytometry analysis of forward scatter-side scatter gating (Fig. 2d), recurrent AITL cells were ubiquitously CD3 positive (Fig. 2e). In addition, many of them expressed CD20 at significant levels (Fig. 2f). This finding was consistent with the results of immunohistochemical analysis. It was strongly suggested that the expression of CD20 on the AITL cell surface began during the second relapse. The expression of other surface markers was as follows; $\mathrm{CD}_{2}^{+}, \mathrm{CD}^{+}, \mathrm{CD}^{+}$, $\mathrm{CD}^{-}, \mathrm{CD} 8^{-}, \mathrm{CD}^{-} 0^{+}, \mathrm{CD} 19^{-}, \kappa$ and $\lambda$. Results of in situ hybridization for EBV latency-associated RNA (EBER) showed no signals in the lymphoma cells, but a few positive cells in the biopsy tissue were observed (Fig. 1m). Karyotypic analysis of the lymph node showed the following abnormality; $51, \mathrm{XY},+\mathrm{X},+4,+5, \operatorname{add}(7)(\mathrm{p} 22)$, add(10)(q22), add (14)(q32), add(19)(p13), der(20)?(14;20)(q11.2;q11.2),+21,+ $21[7] / 46, X Y[4]$. Flow cytometric analysis of bone marrow aspirate revealed an unusual pattern of expression; $\mathrm{CD} 2^{+}$, $\mathrm{CD}^{+}, \mathrm{CD}^{+}, \mathrm{CD}^{+}, \mathrm{CD}^{+} 0^{+}, \mathrm{CD} 19^{-}, \mathrm{CD} 20^{+}, \kappa$ and $\lambda$. Karyotypic analysis showed the $14 \mathrm{q} 32$ abnormality; 52,XY,+X,del (1)(p?), $+3,+5, \operatorname{add}(7)(\mathrm{p} 22), \operatorname{add}(10)(\mathrm{q} 22), \operatorname{add}(14)(\mathrm{q} 32), \operatorname{add}(19)$ (p13),+21,+21, mar1[2]/47,XY,+Y[1]/46,XY[16]. 

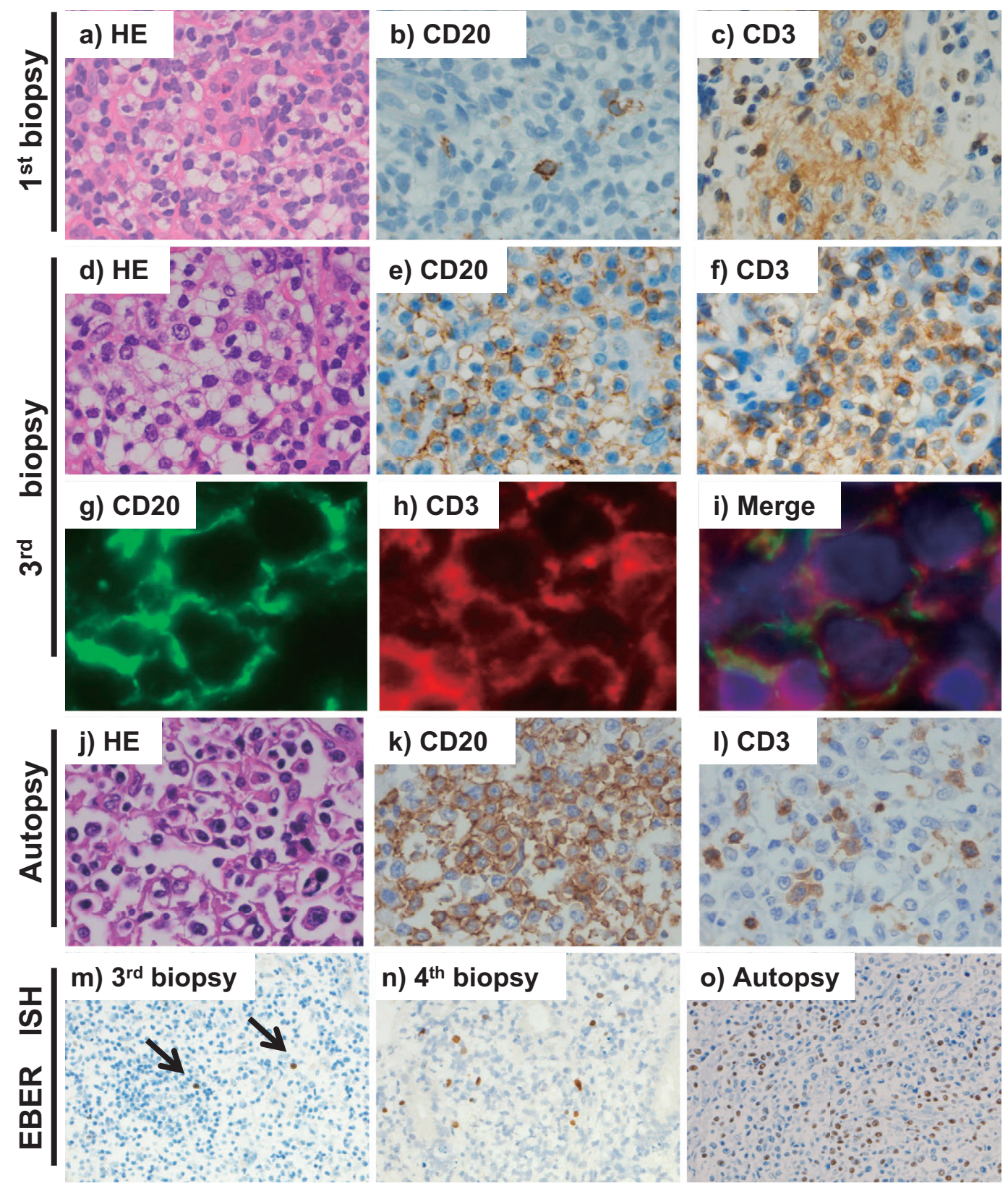

Figure 1. Pathologic examination. (a) First biopsy (inguinal lymph node, Hematoxylin and Eosin staining; January 2006) showed diffuse infiltration of atypical lymphocytes with clear cytoplasm and marked proliferation of arborizing HEV consistent with AITL. (b) These lymphocytes were negative for CD20. (c) These lymphocytes were positive for CD3. (d) Third biopsy (cervical lymph node, Hematoxylin and Eosin staining; January 2008) showed the proliferation of medium- to large-sized lymphocytes with clear cytoplasm reminiscent of the typical AITL cells in the first biopsy and consistent with the features of recurrent lymphoma. (e)(f) These lymphocytes were positive for both CD20 and CD3. (g)-(i) Immunostaining of serial sections of the cervical lymph node in the third biopsy; (g) atypical lymphocytes were diffusely positive for CD20 (green); (h) these lymphocytes were also positive for $\mathrm{CD3}$ (red); (i) merged image of (g) and (h). $\mathrm{CD3}^{+} \mathrm{CD20}^{+}$cells are shown in yellow. (j) Mesenteric lymph node at autopsy (Hematoxylin and Eosin staining; February 2009) showed many lymphocytes with large nuclei and eosinophilic cytoplasm, suggesting the expansion of DLBCL. AITL cells with pale cytoplasm were also infiltrated. (k) Lymphocytes were diffusely positive for CD20. (l) Lymphocytes were slightly positive for CD3. (m)-(o) EBER in situ hybridization; (m) EBER in the third biopsy of recurrent phase (January 2008) showed that a few cells were positive (arrows); (n) EBER in the fourth biopsy at the end stage of disease (January 2009) showed that positive cells were increased in number; (o) EBER at autopsy (February 2009) showed diffuse detection of positive cells supporting the notion that the lesion was overwhelmed by DLBCL cells. 
(a)

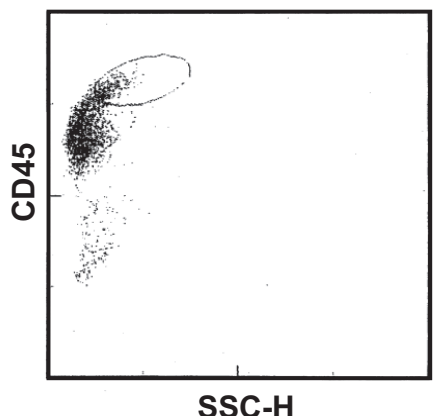

(d)

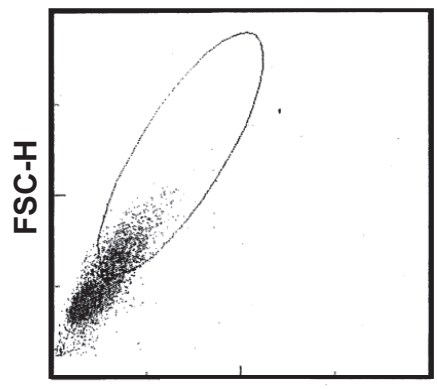

SSC-H (b)

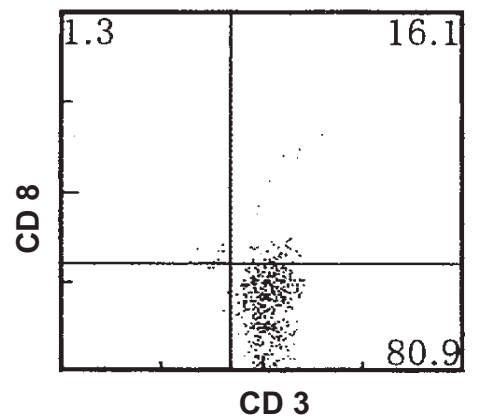

(e)

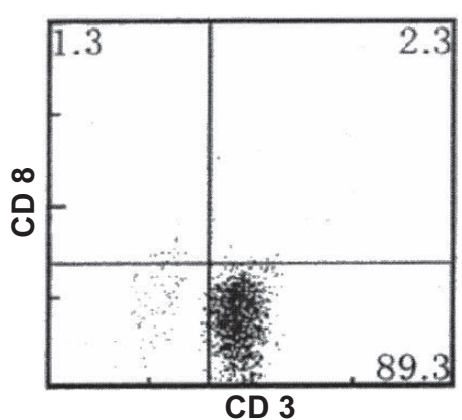

(c)

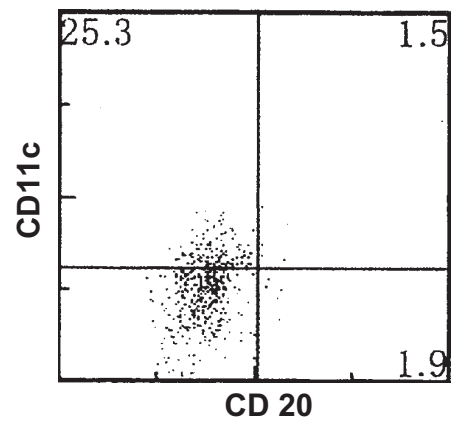

(f)

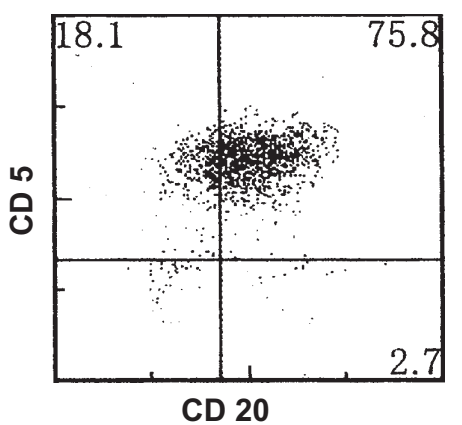

Figure 2. Flow cytometry. (a)-(c) CD45 gating of the axillary lymph node in the second biopsy: abnormal lymphocytes are positive for CD3 and negative for CD20, demonstrating the usual AITL pattern. (d)-(f) Forward scatter-side scatter (FSC-SSC) gating of the cervical lymph node in the third biopsy: abnormal lymphocytes are positive for both CD3 and CD20, strongly suggesting that the majority of AITL cells are double positive.

\section{Fourth biopsy: (AITL with $\mathrm{CD}^{+} \mathrm{CD20}^{+}$, ileocecal ul- cer legion, in January 2009)}

Colorectal fiber-optic examination detected a hemorrhagic ulcer in the ileocecal colon. Pathologic analysis of the ulcerated mucosa biopsy specimen showed diffuse infiltration of lymphoma cells, which were diffusely positive for CD3, a finding consistent with recurrent AITL. These cells were also positive for $\mathrm{CD} 20$, suggesting that an unusual subclone prevailed. However, the specimen was crushed with ulcer debris, and detailed morphologic analysis and flow cytometric analysis therefore were not available. EBER-positive cells were also detected, and the positive cells increased in number (Fig. 1n).

\section{Autopsy: (expansion of B-immunoblasts with CD3 $\mathrm{CD20}^{+}$, in February 2009)}

The autopsy revealed the systemic progression of lymphoma. There were severe bleeding ulcers in the ileocecal and transverse colons to which the lethal hemorrhage was attributable. The lymphoma also involved many other organs, including the para-aortic lymph nodes, subcutaneous soft tissues, and bone marrow. Microscopic investigation of these lesions showed that $\mathrm{CD}^{+}$AITL cells were detectable but were much fewer in number than the $\mathrm{CD} 20^{+}$atypical $\mathrm{B}$ cells with enlarged nuclei and eosinophilic cytoplasm that was diffusely infiltrated (Fig. 1j, k, 1). Many of the Bimmunoblasts were found to be positive for EBV by in situ hybridization (Fig. 10). We concluded that the pathologic diagnosis at the end stage of the disease was a composite of AITL and diffuse large B-cell lymphoma (DLBCL). These findings together with the pattern of repeated relapses of AITL suggest that the immunoblastic proliferation of EBVpositive B cells eventually developed into DLBCL. Because of the prevailing CD3 CD20 ${ }^{+}$DLBCL cells in all tissue lesions at autopsy, we were unable to determine whether $\mathrm{CD} 20^{+}$AITL cells were present or absent at the time of death.

\section{Discussion}

In addition to CD19 and CD79a, CD20 is widely accepted as a B-lineage marker. Most of B-cell lymphoma cells express the CD20 antigen on the cell membrane. Rituximab, a human/mouse chimeric anti-CD20 antibody, is a currently available molecular target-based drug whose clinical prevalence has led to prolonged overall and event-free survival in patients with DLBCL (8). To our knowledge, CD20 is thought to be distinctively negative in AITL (4), and its expression in AITL previously has not been reported.

In the present case, although the AITL cells of the first and the second biopsy specimens were CD20, cells of the 
third biopsy specimens taken during the recurrent stages were simultaneously positive for CD3 and CD20 as identified by immunohistochemical double staining and flow cytometry. An abnormal $\kappa / \lambda$ restriction ratio was not observed in the third biopsy specimen. These findings strongly support the finding of aberrant CD20 expression in AITL cells. Flow cytometry showed $\mathrm{CD} 2^{+}, \mathrm{CD}^{+}, \mathrm{CD}^{+}$, and $\mathrm{CD}^{+}$expression in the lymphoma cells, indicating a decreased possibility of B-cell clonal expansion. Because no signs of EBV were found in lymphoma cells, those were different from the EBV-positive DLBCL clones.

With respect to karyotypic analysis, complex karyotypes including add(14)(q32) were found in the third biopsy specimens with the $14 q 32$ chromosome demonstrating an $\operatorname{IgH}$ gene rearrangement that suggests its association with B-cell clonality. Southern blot analysis was not available. In a recent review of 24 case reports about $\mathrm{CD} 20^{+} \mathrm{T}$-cell malignancies, 10 cases were examined by chromosome analysis (6). No cases showed a $14 \mathrm{q} 32$ abnormality. It is worth mentioning that we did not detect $\operatorname{add}(14)(\mathrm{q} 32)$ in the second biopsy specimen, in which the AITL cells were still negative for CD20 and therefore, we were unable to evaluate $\operatorname{add}(14)(q 32)$ in the present case.

It is well known that AITL and EBV-associated DLBCL sometimes appear as composite lymphoma (9-12). EBVpositive cell clones in AITL may develop B-cell lymphoma and the EBV load is correlated with clinical manifestation of AITL (13). Skugor ND et al reported a case of DLBCL occurring after treatment of AITL and suggested that immunodeficiency after cytotoxic chemotherapy caused EBV reactivation to develop DLBCL (14). Its clinical course is similar to the present case; EBV reactivation was associated with the highly aggressive progression of DLBCL in the last terminal stage. It is supposed that AITL was suppressed naturally along with the course. However, the association of CD20 expression between AITL cells at the third biopsy and EBV-positive DLBCL cells at the autopsy remains unidentified.

Although we were unable to clarify the mechanism and role of aberrant CD20 expression on malignant T-cells, the instability of the CD20 antigen may point to a role as an activation marker $(15,16)$. Therefore, the timing of the administration of rituximab is optimal because CD20 AITL clone would be monoclonal. The administration of rituximab is thought to improve the prognosis of AITL by suppressing EBV-positive B-immunoblasts in the microenvironment, and a clinical study to evaluate the effectiveness of rituximab is ongoing (17).

We present here the first confirmed case of aberrant CD20 expression on AITL cells. It may provide important clues to the pathogenesis and the therapeutic strategy of AITL.
The authors state that they have no Conflict of Interest (COI).

\section{References}

1. Alizadeh AA, Advani RH. Evaluation and management of angioimmunoblastic T-cell lymphoma: a review of current approaches and future strategy. Clin Adv in Hematol \& Oncol 6: 899-909, 2008.

2. Mourad N, Mounier N, Brière J, et al; Groupe d'Etude des Lymphomes de l' Adulte. Clinical, biologic, and pathologic features in 157 patients with angioimmunoblastic T-cell lymphoma treated within the Groupe d'Etude des Lymphomes de l'Adulte (GELA) trials. Blood 111: 4463-4470, 2008.

3. Tan BT, Warnke RA, Arber DA. The frequency of B- and T-cell gene rearrangements and Epstein-Barr virus in T-cell lymphomas: a comparison between angioimmunoblastic T-cell lymphoma and peripheral T-cell lymphoma, unspecified with and without B-cell proliferations. J Mol Diagn 8: 466-475, 2006.

4. Went P, Agostinelli C, Gallamini A, et al. Marker expression in peripheral T-cell lymphoma: a proposed clinical-pathologic prognostic score. J Clin Oncol 24: 2472-2479, 2006.

5. Swerdlow SH, Campo E, Harris NL, et al. WHO classification of tumours of haematopoietic and lymphoid tissues. Lyon, International Agency for Research on Cancer 309-311, 2008.

6. Miyazaki K, Ohsaka M, Suzuki Y, Danbara M, Horie R, Higashihara M. CD20-positive T-cell large granular lymphocyte leukemia: case report and review of the literature. Intern Med 48: 14431447, 2009.

7. Suyama T, Furuya M, Nishiyama M, et al. Up-regulation of interfero gamma (IFN- $\gamma$ )-inducible chemokines INF-inducible T-cell alpha chemoattractant and monokine induced by INF-gamma and of their receptor $\mathrm{CXC}$ receptor 3 in human renal cell carcinoma. Cancer 103: 258-267, 2005.

8. Coiffier B, Lepage E, Briere J, et al. CHOP chemotherapy plus rituximab compared with CHOP alone in elderly patients with diffuse large-B-cell lymphoma. N Engl J Med 346: 235-242, 2002.

9. Xu Y, Mckenna RW, Hoang MP, Collins RH, Kroft SH. Composite angioimmunoblastic T-cell lymphoma and diffuse large B-cell lymphoma: a case report and review of literature. Am J Clin Pathol 118: 848-854, 2002

10. Hawley RC, Cankovic M, Zarbo RJ. Angioimmunoblastic T-cell lymphoma with supervening Epstein-Barr virus-associated large Bcell lymphoma. Arch Pathol Lab Med 130: 1707-1711, 2006.

11. Attygalle AD, Kyriakou C, Dupuis J, et al. Histologic evolution of angioimmunoblastic T-cell lymphoma in consecutive biopsies; clinical correlation and insights into natural history and disease progression. Am J Surg Pathol 118: 1077-1088, 2007.

12. Carbone A, Gloghini A, Dotti G. EBV-associated lymphoproliferative disorders: classification and treatment. Oncologist 13: 577585, 2008.

13. Battegay M, Berger $C$, Rochlitz $C$, et al. Epstein-Barr virus load correlating with clinical manifestation and treatment response in a patient with angioimmunoblastic T-cell lymphoma. Antiviral Ther 9: 453-459, 2004.

14. Skugor ND, Perić Z, Vrhovac R, Radić-Kristo D, Kardum-Skelin I, Jaksić B. Diffuse large B-cell lymphoma in patient after treatment of angioimmunoblastic T-cell lymphoma. Coll Antropol 1: 241-245, 2010.

15. Sun T, Akalin A, Rodacker M, Braun T. CD20 positive T cell lymphoma: is it a real entitiy? J Clin Pathol 57: 442-444, 2004.

16. Rahemtullah A, Longtine JA, Harris NL, et al. CD20+ T-cell lymphoma: clinicopathologic analysis of 9 cases and a review of the literature. Am J Surg Pathol 32: 1593-1607, 2008.

17. Joly B, Frenkel V, Gaulard $P$, et al. Rituximab in combination with CHOP regimen in angioimmunoblastic T-cell lymphoma (AITL). Preliminary results in 9 patients treated in a single institution. Blood 106: 2686, 2005.

(C) 2011 The Japanese Society of Internal Medicine http://www.naika.or.jp/imindex.html 